\title{
GAS LIMITED HYDROGENATION OF 1-OCTENE IN A FIXED BED: UPFLOW VERSUS DOWNFLOW
}

\author{
F. Reynders and W. Nicol \\ Department of Chemical Engineering, University of Pretoria, Pretoria, South Africa
}

\begin{abstract}
Two downflow operational modes with different hydrodynamic states were compared to upflow operation. Various gas limited conditions $(\gamma=15-60)$ were employed. The downflow mode with the higher wetting efficiency consistently outperformed the downflow mode with the lower wetting efficiency. Both the downflow modes outperformed the upflow mode at high gas mass fluxes and underachieved against upflow at lower gas mass fluxes. The results stand in contradiction to the theory on direct gas-solid mass transfer under trickle flow conditions. The switching behavior of the upflow mode performance was attributed to hydrodynamic regime shifts, hinting that the perceived 'poor' performance of upflow reactors under gas limited conditions might be linked to slugging in laboratory scale reactors.
\end{abstract}

KEYWORDS: Catalysis, Hydrodynamics, Mass Transfer, Multiphase Reactors, Packed Bed

\section{INTRODUCTION}

Packed bed multiphase reactors are considered to be one of the workhorses of the refining industry and consequently literature is rife with studies on this class of reactor. The complexity of the hydrodynamics, despite the relative simplicity of this reactor type, is the major reason for the ongoing pursuit in open literature for improved understanding. One of the simple questions frequently addressed when considering the reaction of a gas and liquid reagent on a packed bed of catalyst is whether cocurrent upflow or cocurrent downflow should be employed. This question has been addressed as far back as 1975 (Goto et al). Numerous studies have followed which made it clear that major differences are possible (up/downflow conversion difference of up to $33 \%$ (Khadilkar et al., 1996)) at similar superficial velocities, but no distinct winner emerged, indicating that a straightforward answer to this question is not possible (Mazzarino et al., 1989; Khadilkar et. al., 1996, Beaudry et al., 1987). Contradictory results were especially obtained when comparing low interaction downflow (or trickle flow) with upflow reactors (Mills et al., 1984).

It became apparent that the gas reactant partial pressure and the liquid reactant concentration play a decisive role in determining the optimum configuration and hence the limiting reagent 
concept was introduced to explain the conflicting observations (Beaudry et al., 1987). In essence the unwetted external surface area under low velocity downflow conditions (trickle flow) was seen as either a rate reducer for reactions limited by the liquid phase reagent, or as a rate enhancer for reactions limited by the gas phase reagent. This is due to the fact that gas reactant can be supplied through the dry surface (and wet surface), while liquid reactant can only access the active sites via the wetted surface.

The limiting reagent concept was extended by Khadilkar (1996) where the $\gamma$-factor was introduced to quantify the extent of gas/liquid limitation. The $\gamma$-factor compares the maximum diffusional rates of the different reagents and for 1-ctene hydrogenation it is expressed by:

$$
\gamma=\frac{D_{e, O C T} C_{b u l k, O C T}}{D_{e, H_{2}} C_{H_{2}}^{*}}
$$

Values of $\gamma \gg 1$ and $\gamma \ll 1$ would be indicative of gas- and liquid-limiting reaction systems respectively. In his paper, Khadilkar (1996) applied the criteria to explain the findings of prominent comparison studies and also showed how the same reaction system under different reactant concentration ratios results in a switch between upflow/downflow performances. In essence gas limited reactions (according to the $\gamma$-factor) favoured downflow operation, while the opposite was found for liquid limited reactions. The relative simple $\gamma$-criterion has since been used by some as a standard for deciding upon the preferred mode of operation. The underlying assumption in doing this is that direct gas-solid mass transfer dominates the overall reaction rate for gas limited reactions.

Recently the importance of the hydrodynamic state under trickle flow conditions have been accentuated (Loudon et al., 2006). It has been shown in 2 studies (van der Merwe and Nicol, 2008; van Houwelingen et al., 2011) that severe difference in reactor performance is possible when employing different prewetting techniques. Since these differences are associated with different external wetting efficiencies (van Houwelingen, 2006), the dry surface effect as explained by the limiting reagent concept will affect the overall reaction rate. In the paper by van Houwelingen (2011) the trend with regards to liquid-limited reactions was in accordance to the expected outcome, where the downflow hydrodynamic state with the highest wetting efficiency performed better than the lower wetting counterpart, but worse than the upflow configuration.

It was however shown that the rate enhancement was a joint contribution from improved wetting as well as improved liquid-solid mass transfer. This highlights the fact that the $\gamma$-factor does not distinguish between mass transfer differences caused by hydrodynamic variations. The extension of the conventional up/down comparison to an up/down/down comparison used in this paper is useful in terms of providing additional insight into the effect of external wetting fraction on reactor performance.

In this study the above mentioned ternary comparison was applied to a gas limited reaction. The reaction system is identical to the system described by van Houwelingen (2011). The reactor 
pressure, hydrogen partial pressure and 1-octene feed concentration was modified to ensure gas limited conditions.

\section{EXPERIMENTAL}

For a detailed discussion on the experimental setup, see van Houwelingen (2011). Figure 1 gives a schematic of the reactor setup. The reactor has an inside diameter of $50 \mathrm{~mm}$ and is $1 \mathrm{~m}$ long (9 $\mathrm{cm}$ layer of $110 \mathrm{~g})$ of active catalyst $\left(\rho_{\text {cat }}=1100 \mathrm{~kg} / \mathrm{m}^{3}\right)$ wedged between two $45 \mathrm{~cm}$ layers of inert $3 \mathrm{~mm}$ alumina support).

The pretreatment or prewetting procedures of the two downflow modes are identical to that of van Houwelingen (2011). The extensively prewetted mode represents the upper hysteresis branch, while the Levec-prewetted mode represents the lower hysteresis branch. The conditions employed for each of the operational modes are given in Table 1. Each feed condition is denoted as a run number and entails 12 steady state readings (4 liquid velocities and 3 operational modes). In addition random repeat experiments were performed.

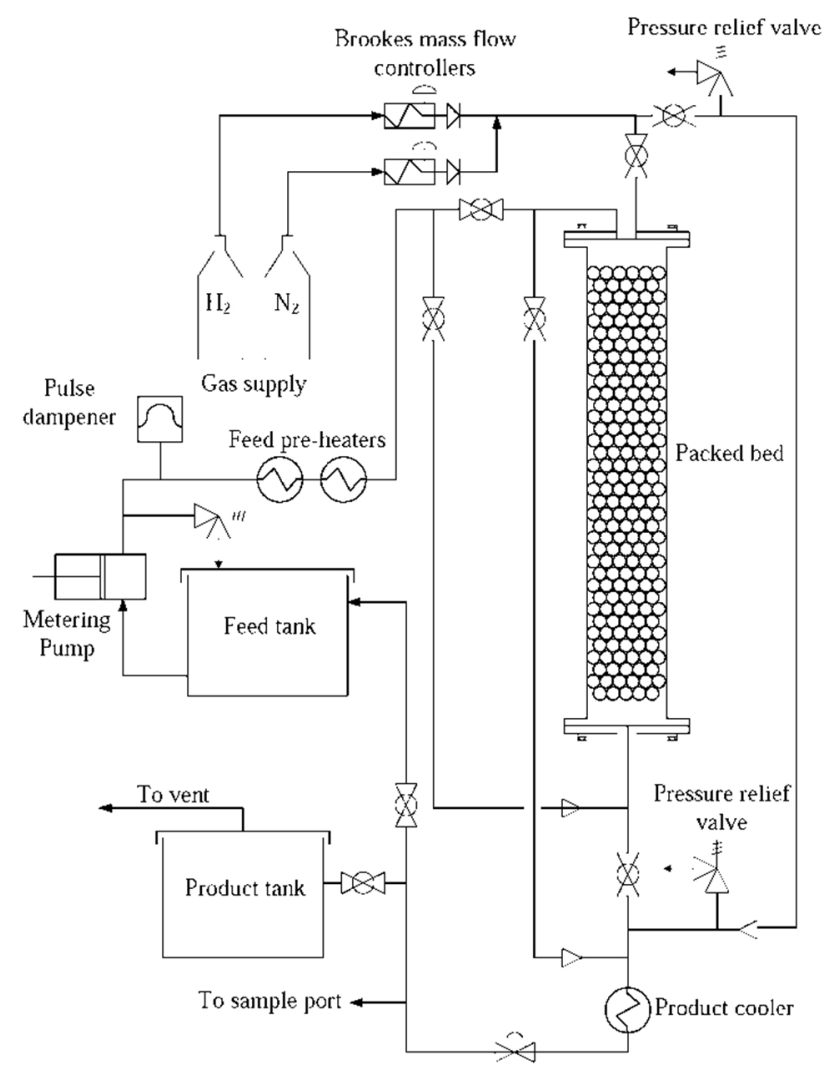

Figure 1: PBR experimental setup 


\section{RESULTS AND DISCUSSION}

The average reaction rates data for all four runs are given in figure 2. Due to the gas limitation the conversion-reactor depth profile is expected to be linear and accordingly the reaction rate at any position in the bed will be similar to the average reaction rate. From figure 2 it is evident that the average reaction rate increase as the hydrogen partial pressure increase. The $\gamma$-factor was determined for all the experimental runs based on the inlet conditions, estimated diffusivities and predetermined hydrogen solubility and therefore only gives a relative indication of the extend of gas limitation. The primary observation from the data is the inconsistent position of the upflow results relative to the two downflow modes. The initial guess, based on the contribution of the gas-solid transport step on dry catalyst area, would have predicted the reaction rate for all the upflow runs to be lower than that of the downflow runs, but this was not found to be the case. Furthermore it is interesting to note that the results from the Levec prewetted mode is consistently lower than the extensively wetted counterpart. This contradicts the accepted enhancement effect that the direct gas-solid mass transfer is thought to have since the Levec prewetted mode has a higher fraction of unwetted catalyst surface.

Table 1: Experimental conditions for all 3 operational modes

\begin{tabular}{ccccc}
\hline Reaction system & 1 & 2 & 3 & 4 \\
\hline$\gamma^{*}$ & 60 & 30 & 15 & 15 \\
$\rho_{G}\left(\mathrm{~kg} / \mathrm{m}^{3}\right)$ & 5.2 & 4.2 & 2.3 & 5.3 \\
$P_{T o t}(\mathrm{bar})$ & 6 & 6 & 6 & 9 \\
$v_{G}(\mathrm{~mm} / \mathrm{s})$ & 16 & 16 & 16 & 16 \\
$M_{G}(\mathrm{~g} / \mathrm{mole})$ & 24 & 19 & 11 & 16 \\
$p_{H_{2}}(\mathrm{bar})$ & 0.9 & 2 & 4 & 4 \\
$m_{G}(\mathrm{~g} / \mathrm{s})$ & 0.16 & 0.13 & 0.07 & 0.17 \\
\hline
\end{tabular}

*Based on predetermined $\mathrm{He}$ of $2.2 \mathrm{~mole} / \mathrm{m}^{3} \mathrm{bar}$ at $60^{\circ} \mathrm{C}$, and diffusivities estimated according to the Wilke-Chang correlation

Runs 3 and 4 are of particular interest where the same $\gamma$-factor was employed under different total pressure conditions. The downflow modes exhibit similar results, while the upflow switches from above the extensively prewetted mode in run 3 to below the Levec mode in run 4 . This indicates that the gas density plays a vital role in the performance of the upflow mode, while its effect on the downflow modes is negligible. The upflow trend for run 1 is similar to run 4, both at relatively high gas densities. This observation can be related to the prevailing hydrodynamic regime in the bed. Correlations suggest that both downflow modes will be in the low interaction regime irrespective of the gas density (Larachi et al., 1991), while a regime switch might be possible for the upflow mode (packed bubble column). The regime mappings suggested by Murugesan and Sivakumar (2002) and Gianetto and Specchia (1992) was used to map the upflow operating conditions (figure 3 ). 
Since the observations cannot solely be linked to the difference in external wetting a closer examination of the external transport steps should be performed. A reactor model with an associated choice of complexity should be used to perform the analysis. The major factors affecting the outcome of the model will be the choice of mass transfer coefficients, wetting efficiencies and the extend of internal diffusion considerations. With regards to mass transfer coefficients and wetting efficiency the variability of accepted correlations is a concern. This concern can be partially bypassed by using the liquid-solid mass transfer and wetting efficiencies determined by van Houwelingen (2011) in the same reactor employing the same catalyst and inert packing under different operating conditions. The handling of the internal diffusional aspects is the major issue of the modeling approach. This should preferably not be oversimplified due the dry-side contribution effect used to explain superior performance for downflow gas limited reactions. Although not reported in this paper, detailed slurry kinetic experiments (employing whole particles) were performed on the 1-octene reaction in the absence of any external mass transport effects (Reynders, 2011). The obtained particle kinetics had a clear dependency on both 1-octene and hydrogen concentration and a Langmuir-Hinshelwood rate form with two additional adsorption coefficients had to be used to fit the kinetic data since a simple powerlaw relationship did not suffice. This severely complicates the intraparticle diffusion analysis since no simplified model is available to estimate the overall particle rate at partially wetted conditions. To bypass the problem, the assumption was made that the major differences in experimental results are linked to external transport differences. The particle kinetics in the packed bed reactor was merely reduced to slurry particle kinetics multiplied with the estimated wetting efficiency for each mode (note that eggshell catalyst is used). Using a binary concentration (1-octene and hydrogen) plug flow model with all the associated mass transfer steps, the gas-liquid transfer coefficient was varied to fit the experimental data. Although vastly different gas-liquid transfer coefficients had to be employed to fit the upflow data (volumetric values varied between 0.01 to $0.08 \mathrm{~s}^{-1}$ for upflow and between 0.02 and $0.04 \mathrm{~s}^{-1}$ for downflow), it was evident that the liquid-solid boundary layer gradient of both the hydrogen an 1-octene concentration was very small for all the runs, suggesting that gas-liquid mass transfer is the rate limiting step if the oversimplified particle rate model is used.

The analysis suggest major jumps in the gas-liquid mass transfer coefficient for the upflow experiments of the different runs, while almost constant coefficient values were used for the downflow runs. Major variations in the gas-liquid transfer step for upflow experiments can be attributed to a flow regime shift where the lower coefficient values relates to the slug flow regime, characterized by limited gas-liquid interfacial area. Using the available regime maps for packed bubble columns (Figure3) it is evident that slug flow might occur (more likely for the Gianetto mapping). The reported studies in the paper of Khadilkar (1996) were also mapped and it is interesting to note the close vicinity of some studies to the slugging regime. The poor performance of the upflow results in these studies might be linked to sluggish gas-liquid mass transfer in the slug flow regime, rather than the complete wetting characteristics. The claim can 


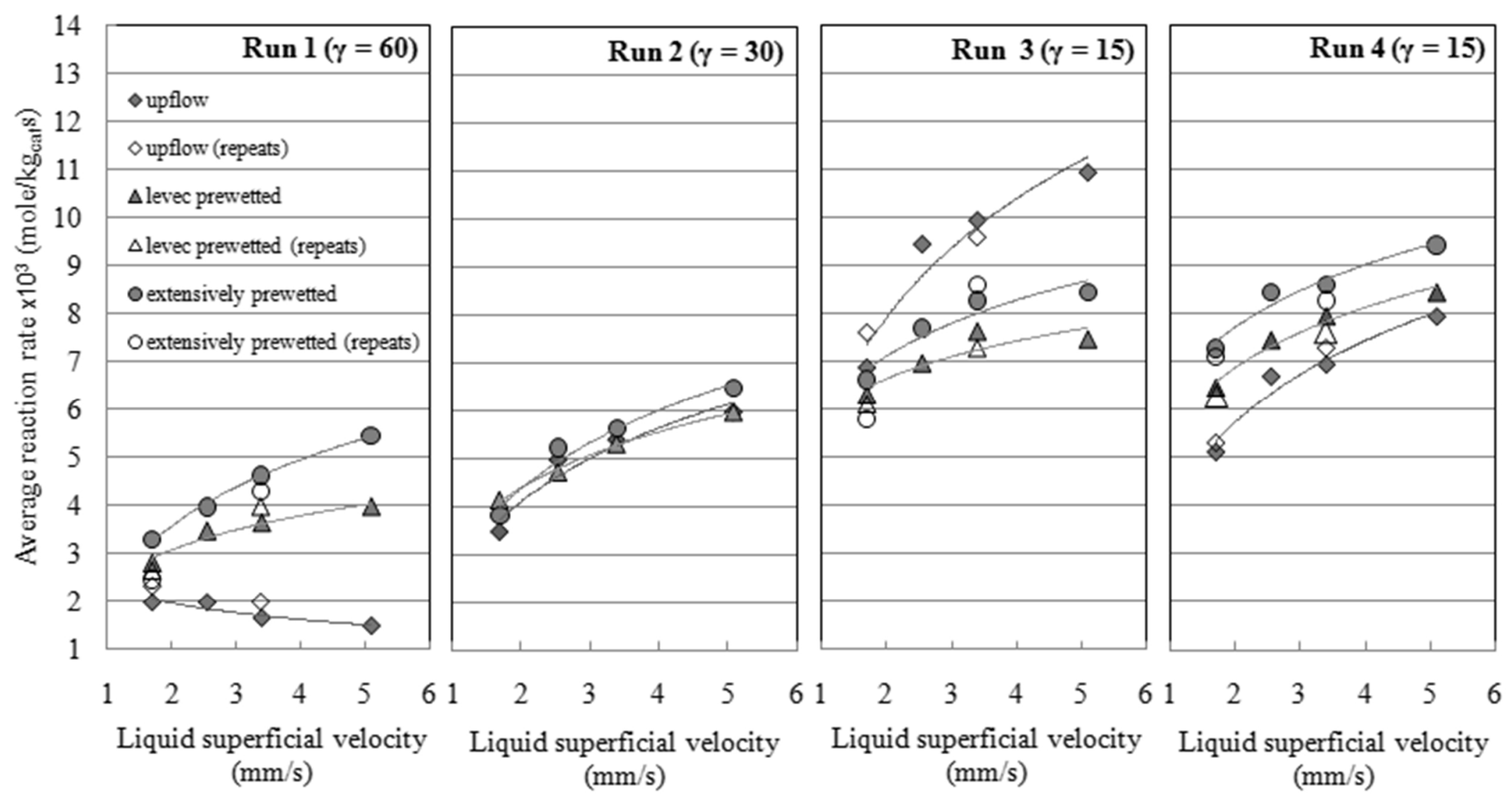

Figure 2: Experimental results 

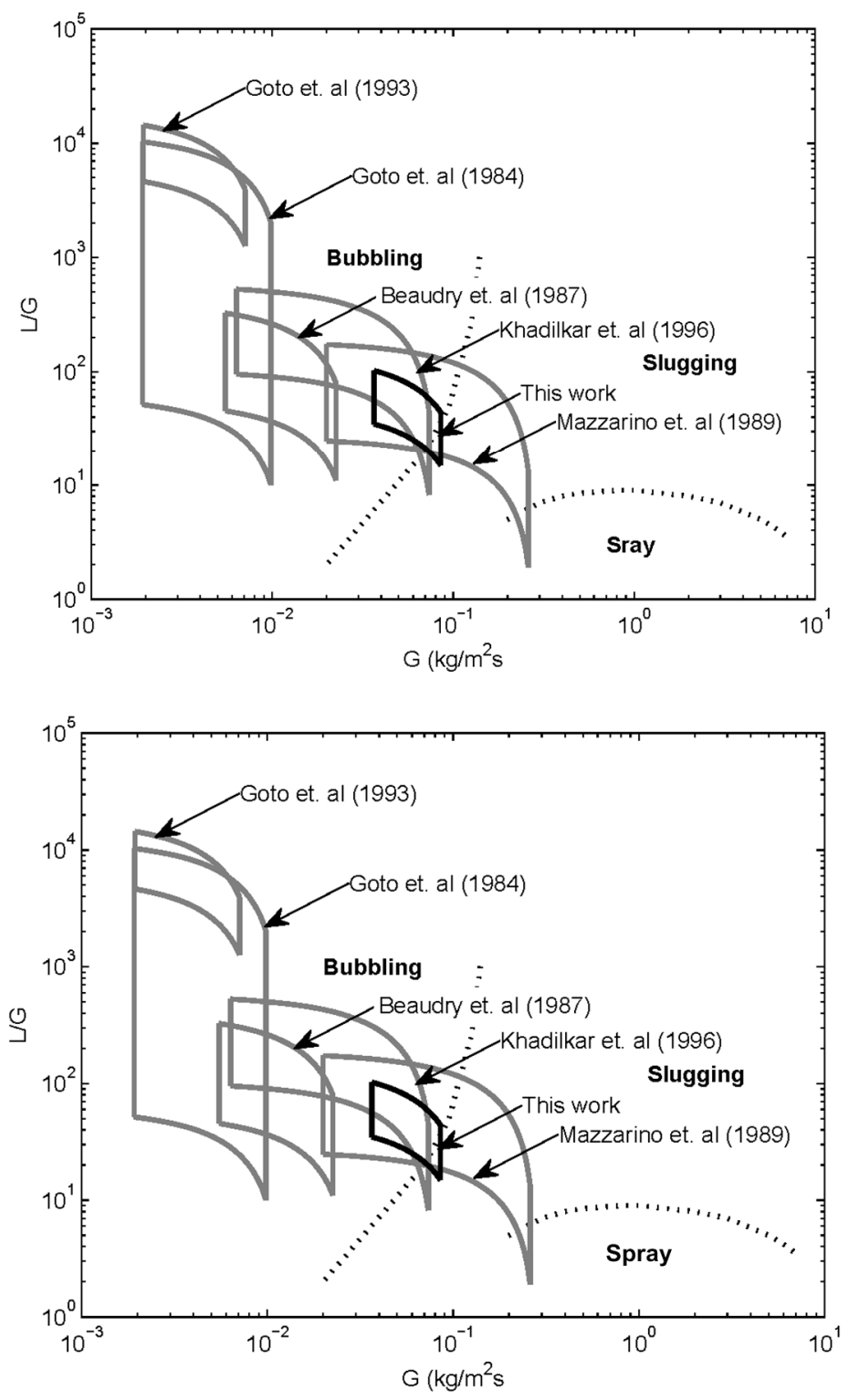

Figure 3: Packed bubble column regime mappings from (top) Gianetto and Specchia (1992) and (bottom) Murugesan and Sivakumar (2003) 
however only be substantiated with detailed modeling and fully characterized transport coefficients.

\section{CONCLUSIONS}

The results obtained for the gas limited hydrogenation of 1-octene do not provide any evidence that direct gas-solid mass transfer enhances the overall reaction rate. The highest wetting downflow mode consistently outperformed the lower wetting downflow mode, while the upflow mode behaved in an inconsistent manner. The results clearly indicate that gas limited reactions are not necessarily favoured by downflow operation and the interpretation of Kadilkar (1996) cannot be applied generically to all gas limited reactions. The results of this specific study show that external transport differences are likely to reverse the suggested trend of downflow superiority for gas limited reactions. The inconsistent behaviour of the upflow operation was linked to a possible switch from the bubbling to the slugging regime, an explanation that might also hold for the interpretation of prior gas limited studies. The results highlight the importance of the liquid-side mass transfer steps and its dependency on the operating regime.

\section{NOTATION}

$C_{\text {bulk,OCT }}$

$C_{H_{2}}^{*}$

$D_{e, H_{2}}$

$D_{e, O C T}$

$D_{c}$

$d_{p}$

$F r_{i}$

G

He

$L$

$m_{G}$

$P_{\text {Tot }}$

$v_{G}$

$v_{L}$

$p_{H_{2}}$

\section{Greek letters}

Bulk 1-octene concentration

Saterated hydrogen concentration in liquid phase

Effective diffusivity of dissolved hydrogen, $\mathrm{m}^{2} / \mathrm{s}$

Effective diffusivity of 1-octene, $\mathrm{m}^{2} / \mathrm{s}$

Column diameter, $\mathrm{m}$

Particle diameter, $\mathrm{m}$

Froude number of phase $i,=\frac{v_{i}^{2}}{g d_{p}}$

Gas mass flux, $\mathrm{kg} / \mathrm{m}^{2} \mathrm{~s}$

Henry's law constant for hydrogen, mole $/ \mathrm{m}^{3}$ bar

Liquid mass flux, $\mathrm{kg} / \mathrm{m}^{2} \mathrm{~s}$

Gas mass flow rate, $\mathrm{kg} / \mathrm{s}$

Total system pressure, bar

Gas superficial velocity, $\mathrm{mm} / \mathrm{s}$

Liquid superficial velocity, $\mathrm{mm} / \mathrm{s}$

Hydrogen partial pressure, bar
$\varepsilon$
Bed porosity
$\gamma$
$=\frac{D_{e, O C T} C_{\text {bulk }, O C T}}{D_{e, H_{2}} C_{H_{2}}^{*}}$
$\rho$
Gas density, $\mathrm{kg} / \mathrm{m}^{3}$ 


$\begin{array}{ll}\psi & =\frac{\sigma_{w}}{\sigma_{L}}\left(\frac{\mu_{w}}{\mu_{w}}\left(\frac{\rho_{L}}{\rho_{L}}\right)^{2}\right)^{0.33} \\ \mu & \text { Viscosity, Pa.s } \\ \sigma & \text { Surface tension, N/m }\end{array}$

\section{REFERENCES}

(1.) Beaudry, E. G.; Dudukovic, M. P. and Mills, P. L., 1987, AlChE Journal, 33 (9), 1435 1447.

(2.) Doraiswamy, L.K. and Sharma, M.M., Heterogeneous reactions, Vol.I, John Wiley ans Sons, New York, 1984

(3.) Dudukovic, M. P.; Larachi, F. and Mills, P. L., 2002, Catalysis reviews, 44 (1), 123 -246.

(4.) Gianetto, A. and Specchia, V., 1992, Chemical Engineering Science, 47 (13), 3197 - 3213.

(5.) Goto, S. and Mabuchi, K., 1984, The Canadian Journal of Chemical Engineering, 62, 865 $-868$

(6.) Goto, S.; Chatani, T. and Matouq, M. H., 1993, The Canadian Journal of Chemical Engineering, 71, $821-823$.

(7.) Goto, S.; Levec, J. and Smith M.J., 1975, Industrial and Engineering Chemistry, Process Design and Development, 14 (4),473 - 478.

(8.) Khadilkar, M. R.; Wu, Y. X. and Dudukovic, M. P., 1996, Chemical Engineering Science, 51 (10), $2139-2148$.

(9.) Larachi, F.; Laurent, A.; Midoux, M. and Wild, G., 1991, Chemical Engineering Science, $46(5 / 6), 1233-1246$

(10.) Loudon, D.; van der Merwe, W. and Nicol, W., 2006, Chemical Engineering Science, 61, $7551-7562$

(11.) Mazzarino, I.; Occhetti, M.; Baldi, G. and Sicardi, S., 1989, Chemical Engineering Communication, 75, $225-240$

(12.) Mills, P.L.; Beaudry, E.G and Dudukovic, M.P., 1984, I.Chem.E Symposium, 87, 526 535

(13.) Murugesan, T. and Sivakumar, V., 2002, Chemical Engineering Journal, 88, 233 - 243.

(14.) Reynders, F., 2011, Online MSc Dissertation, http://upetd.up.ac.za/ETD-db/ETDsearch/search

(15.) Van der Merwe, W. and Nicol, W, 2008, AlChE Journal, 54(10), 249 - 257

(16.) van Houwelingen, A. J.; Sandrock, C. and Nicol, W., 2006, AlChE Journal, 52 (10), 3532 -3542 .

(17.) Van Houwelingen, A.J.; Kok, S., and Nicol, W., 2011, AlChE Journal, n/a. doi: 10.1002/aic. 12342

(18.) Van Houwelingen, A.J.; Sandrock, C. and Nicol, W., 2006, AlChE Journal, 52(10), 3532 3542 